\title{
Pesticide Use Trends in the U.S.: a 20-Year U.S. Summary 1
}

Frederick M. Fishel ${ }^{2}$

\section{Introduction}

The EPA, in cooperation with the USDA and FDA, is responsible for regulating the production and use of pesticides in the U.S. This document provides data on volumes used and sales of pesticides from EPA survey data available for the 20-year period, $1987-2001$. The intent of this information is only to present an objective profile and does not attempt to interpret, reach conclusions about, or make inferences regarding the data. Conclusions should not be drawn in regards to impacts on human health, the environment, or the economy.

\section{Data sources}

The data reported in this document are based upon EPA estimates. EPA does not have a program devoted specifically to estimating pesticide use; rather, they use the best available information from the public domain and proprietary sources. The data are approximate values and not statistically precise. The sources that EPA consults for compiling this information include:
- The Pesticide Data Center in the Biological and Economic Analysis Division of EPA's Office of Pesticide Programs;

- Several national database services for compiling agricultural pesticide use data, including the USDA; and

- Proprietary data sources with vendor permission, including Doane Marketing Research, Inc., Kline and Company, Inc., SRI, Inc., Wood Mackenzie; and Mike Bukley, Inc.

\section{Explanation of data components}

The data presented in the tables separate broad classes of pesticides - herbicides, insecticides, fungicides, and other pesticides. The "herbicide" data combine plant growth regulators (PGR) with them, while "fungicides" and "other" pesticides are presented as combined data and include sulfur, petroleum oil, nematicides, fumigants, rodenticides, molluscicides, aquatic, and pesticides for the control of birds and fish. Data for wood preservatives, specialty biocides, and chlorine/hypochlorites are not

1. This document is PI-142, one of a series of the Pesticide Information Office, Florida Cooperative Extension Service, Institute of Food and Agricultural Sciences, University of Florida. Original publication date January 2007. Visit the EDIS Web Site at http://edis.ifas.ufl.edu.

2. Frederick M. Fishel, associate professor, Agronomy Department, and director, Pesticide Information Office; Florida Cooperative Extension Service, Institute of Food and Agricultural Sciences, University of Florida, Gainesville, FL 32611.

The use of trade names in this publication is solely for the purpose of providing specific information. UF/IFAS does not guarantee or warranty the products named, and references to them in this publication does not signify our approval to the exclusion of other products of suitable composition. Use pesticides safely. Read and follow directions on the manufacturer's label.

The Institute of Food and Agricultural Sciences (IFAS) is an Equal Opportunity Institution authorized to provide research, educational information and other services only to individuals and institutions that function with non-discrimination with respect to race, creed, color, religion, age, disability, sex, sexual orientation, marital status, national origin, political opinions or affiliations. U.S. Department of Agriculture, Cooperative Extension Service, University of Florida, IFAS, Florida A. \& M. University Cooperative Extension Program, and Boards of County Commissioners Cooperating. Larry Arrington, Dean 
included in the tables under any pesticide class.

Totals may not add precisely due to rounding. In reporting the amount used, data contained in Tables 5

- 8 are presented as pounds of active ingredient (a.i.)

per acre. The data presented in the "other" pesticide category are for miscellaneous pesticides including nematicides, fumigants, sulfur, petroleum, and other chemicals used for pesticides, such as sulfuric acid and insect repellents. These data represent the combined pesticide usage in the major markets of agriculture, home and garden - which include pesticides applied by homeowners, both indoors and outdoors. These two market sectors do not include pesticide applications by professional applicators. Industrial/commercial/governmental uses involve pesticides applied by professional applicators to industrial, commercial, and governmental facilities; plus custom/commercial applications to homes and gardens, including lawns.

Tables $6-8$ present total volumes of pesticides applied by market sector.

\section{Additional information}

Kiely, T., D. Donaldson, and A. Grube. 2004. Pesticides Industry Sales and Usage: 2000 and 2001 Market Estimates. EPAs Biological and Economic Analysis Division, Office of Pesticide Programs, and Office of Prevention, Pesticides, and Toxic Substances.

http://www.epa.gov/pesticides. 
Pesticide Use Trends in the U.S.: a 20-Year U.S. Summary

Table 1. U.S. pesticide expenditures, all market sectors.

\begin{tabular}{||c|c|c|c|c||}
\hline \hline \multirow{2}{*}{ Year } & \multicolumn{3}{|c||}{ Expenditure (Millions of Dollars) } \\
\cline { 2 - 4 } & Herbicides/PGR & Insecticides & $\begin{array}{c}\text { Fungicide and } \\
\text { Other }\end{array}$ & Total \\
\hline 2001 & 6,410 & 3,124 & 1,556 & 11,090 \\
\hline 2000 & 6,365 & 3,129 & 1,671 & 11,165 \\
\hline 1999 & 6,368 & 3,046 & 1,741 & 11,155 \\
\hline 1998 & 6,853 & 2,872 & 1,691 & 11,416 \\
\hline 1997 & 6,846 & 2,957 & 1,528 & 11,331 \\
\hline 1996 & 6,599 & 2,849 & 1,521 & 10,969 \\
\hline 1995 & 6,276 & 3,017 & 1,488 & 10,781 \\
\hline 1994 & 5,944 & 2,722 & 1,408 & 10,074 \\
\hline 1993 & 5,094 & 2,479 & 1,259 & 8,832 \\
\hline 1992 & 5,004 & 2,198 & 1,183 & 8,385 \\
\hline 1991 & 4,682 & 2,139 & 1,223 & 8,044 \\
\hline 1990 & 4,473 & 2,083 & 1,171 & 7,727 \\
\hline 1989 & 4,305 & 1,978 & 1,141 & 7,424 \\
\hline 1988 & 4,121 & 1,964 & 1,190 & 7,275 \\
\hline 1987 & 3,973 & 2,008 & 1,049 & 7,030 \\
\hline 1986 & 3,858 & 1,759 & 967 & 6,584 \\
\hline 1985 & 3,920 & 1,823 & 963 & 6,706 \\
\hline 1984 & 4,488 & 1,809 & 708 & 7,005 \\
\hline 1983 & 3,870 & 2,074 & 731 & 6,675 \\
\hline 1982 & 3,772 & 2,014 & 540 & 6,326 \\
\hline & & & & \\
\hline
\end{tabular}

Table 2. U.S. pesticide expenditures, agricultural sector.

\begin{tabular}{||c|c|c|c|c||}
\hline \hline \multirow{2}{*}{ Year } & \multicolumn{3}{|c||}{ Expenditure (Millions of Dollars) } \\
\cline { 2 - 4 } & Herbicides/PGR & Insecticides & $\begin{array}{c}\text { Fungicide and } \\
\text { Other }\end{array}$ & Total \\
\hline 2001 & 4,987 & 1,326 & 1,091 & 7,404 \\
\hline 2000 & 5,007 & 1,411 & 1,194 & 7,612 \\
\hline 1999 & 5,012 & 1,370 & 1,243 & 7,625 \\
\hline 1998 & 5,632 & 1,427 & 1,209 & 8,268 \\
\hline 1997 & 5,610 & 1,551 & 1,124 & 8,285 \\
\hline 1996 & 5,399 & 1,480 & 1,128 & 8,007 \\
\hline 1995 & 5,112 & 1,607 & 1,107 & 7,826 \\
\hline 1994 & 4,008 & 1,293 & 1,036 & 7,137 \\
\hline 1993 & 3,987 & 1,123 & 895 & 6,005 \\
\hline 1992 & 3,915 & 1,058 & 829 & 5,802 \\
\hline 1991 & 3,644 & 687 & 884 & 5,215 \\
\hline 1990 & 3,463 & 1,067 & 842 & 5,372 \\
\hline 1989 & 3,255 & 978 & 800 & 5,033 \\
\hline 1988 & 3,080 & 1,010 & 775 & 4,865 \\
\hline 1987 & 2,935 & 1,145 & 650 & 4,730 \\
\hline 1986 & 2,775 & 914 & 600 & 4,289 \\
\hline 1985 & 2,900 & 990 & 615 & 4,505 \\
\hline
\end{tabular}


Pesticide Use Trends in the U.S.: a 20 -Year U.S. Summary

Table 2. U.S. pesticide expenditures, agricultural sector.

\begin{tabular}{||c|c|c|c|c||}
\hline \hline \multirow{2}{*}{ Year } & \multicolumn{3}{|c||}{ Expenditure (Millions of Dollars) } \\
\cline { 2 - 5 } & Herbicides/PGR & Insecticides & $\begin{array}{c}\text { Fungicide and } \\
\text { Other }\end{array}$ & Total \\
\hline 1984 & 3,390 & 418 & 4,711 \\
\hline 1983 & 2,800 & 903 & 450 & 4,511 \\
\hline 1982 & 2,465 & 1,261 & 268 & 3,842 \\
\hline \hline
\end{tabular}

Table 3. U.S. pesticide expenditures, industry/commercial/government sector.

\begin{tabular}{|c|c|c|c|c|}
\hline \multirow[t]{2}{*}{ Year } & \multicolumn{4}{|c|}{ Expenditure (Millions of Dollars) } \\
\hline & Herbicides/PGR & Insecticides & $\begin{array}{c}\text { Fungicide and } \\
\text { Other }\end{array}$ & Total \\
\hline 2001 & 792 & 510 & 233 & 1,535 \\
\hline 2000 & 762 & 468 & 255 & 1,485 \\
\hline 1999 & 794 & 463 & 289 & 1,546 \\
\hline 1998 & 728 & 425 & 292 & 1,445 \\
\hline 1997 & 743 & 386 & 214 & 1,343 \\
\hline 1996 & 721 & 458 & 208 & 1,387 \\
\hline 1995 & 700 & 527 & 202 & 1,429 \\
\hline 1994 & 679 & 533 & 197 & 1,409 \\
\hline 1993 & 660 & 406 & 191 & 1,257 \\
\hline 1992 & 648 & 378 & 186 & 1,212 \\
\hline 1991 & 616 & 328 & 176 & 1,120 \\
\hline 1990 & 593 & 307 & 169 & 1,069 \\
\hline 1989 & 630 & 317 & 180 & 1,127 \\
\hline 1988 & 600 & 394 & 240 & 1,234 \\
\hline 1987 & 576 & 330 & 210 & 1,116 \\
\hline 1986 & 642 & 316 & 192 & 1,150 \\
\hline 1985 & 600 & 315 & 180 & 1,095 \\
\hline 1984 & 720 & 365 & 150 & 1,235 \\
\hline 1983 & 720 & 288 & 144 & 1,152 \\
\hline 1982 & 852 & 305 & 142 & 1,299 \\
\hline
\end{tabular}

Table 4. U.S. pesticide expenditures, home and garden sector.

\begin{tabular}{||c|c|c|c|c||}
\hline \hline \multirow{2}{*}{ Year } & \multicolumn{3}{|c||}{ Expenditure (Millions of Dollars) } \\
\cline { 2 - 5 } & Herbicides/PGR & Insecticides & $\begin{array}{c}\text { Fungicide and } \\
\text { Other }\end{array}$ & Total \\
\hline 2001 & 631 & 1,288 & 232 & 2,151 \\
\hline 2000 & 596 & 1,250 & 222 & 2,068 \\
\hline 1999 & 562 & 1,213 & 209 & 1,984 \\
\hline 1998 & 493 & 1,020 & 190 & 1,703 \\
\hline 1997 & 493 & 1,020 & 190 & 1,703 \\
\hline 1996 & 479 & 910 & 185 & 1,574 \\
\hline 1995 & 465 & 883 & 179 & 1,527 \\
\hline \hline
\end{tabular}


Pesticide Use Trends in the U.S.: a 20-Year U.S. Summary

Table 4. U.S. pesticide expenditures, home and garden sector.

\begin{tabular}{||c|c|c|c|c||}
\hline \hline \multirow{2}{*}{ Year } & \multicolumn{3}{|c|}{ Expenditure (Millions of Dollars) } \\
\cline { 2 - 5 } & Herbicides/PGR & Insecticides & $\begin{array}{c}\text { Fungicide and } \\
\text { Other }\end{array}$ & Total \\
\hline 1994 & 456 & 895 & 175 & 1,526 \\
\hline 1993 & 446 & 870 & 174 & 1,490 \\
\hline 1992 & 441 & 762 & 168 & 1,371 \\
\hline 1991 & 423 & 724 & 162 & 1,309 \\
\hline 1990 & 417 & 710 & 160 & 1,287 \\
\hline 1989 & 420 & 683 & 161 & 1,264 \\
\hline 1988 & 441 & 601 & 175 & 1,217 \\
\hline 1987 & 462 & 534 & 189 & 1,185 \\
\hline 1986 & 441 & 529 & 175 & 1,145 \\
\hline 1985 & 420 & 518 & 168 & 1,106 \\
\hline 1984 & 378 & 542 & 140 & 1,060 \\
\hline 1983 & 350 & 525 & 137 & 1,012 \\
\hline 1982 & 445 & 600 & 130 & 1,175 \\
\hline \hline
\end{tabular}

Table 5. U.S. pesticide amounts used, all market sectors.

\begin{tabular}{||c|c|c|c|c|c||}
\hline \hline \multirow{2}{*}{ Year } & \multicolumn{5}{|c||}{ Million Pounds of Active Ingredient } \\
\cline { 2 - 6 } & Herbicides/PGR & Insecticides & Fungicides & Other & Total \\
\hline 2001 & 553 & 105 & 73 & 472 & 1,203 \\
\hline 2000 & 542 & 122 & 74 & 496 & 1,234 \\
\hline 1999 & 534 & 126 & 79 & 505 & 1,244 \\
\hline 1998 & 555 & 103 & 86 & 462 & 1,206 \\
\hline 1997 & 568 & 112 & 81 & 467 & 1,228 \\
\hline 1996 & 578 & 116 & 79 & 456 & 1,229 \\
\hline 1995 & 556 & 125 & 77 & 452 & 1,210 \\
\hline 1994 & 583 & 124 & 79 & 443 & 1,229 \\
\hline 1993 & 527 & 115 & 80 & 440 & 1,162 \\
\hline 1992 & 554 & 116 & 81 & 435 & 1,186 \\
\hline 1991 & 546 & 114 & 86 & 408 & 1,154 \\
\hline 1990 & 564 & 121 & 91 & 425 & 1,201 \\
\hline 1989 & 567 & 123 & 98 & 405 & 1,193 \\
\hline 1988 & 557 & 132 & 99 & 403 & 1,191 \\
\hline 1987 & 532 & 141 & 100 & 402 & 1,175 \\
\hline 1986 & 590 & 151 & 109 & 416 & 1,266 \\
\hline 1985 & 611 & 161 & 110 & 422 & 1,304 \\
\hline 1984 & 634 & 173 & 109 & 429 & 1,345 \\
\hline 1983 & 573 & 185 & 115 & 435 & 1,308 \\
\hline 1982 & 620 & 198 & 117 & 447 & 1,382 \\
\hline \hline
\end{tabular}


Pesticide Use Trends in the U.S.: a 20-Year U.S. Summary

Table 6. U.S. pesticide amounts used, agricultural sector.

\begin{tabular}{||c|c|c|c|c|c||}
\hline \hline \multirow{2}{*}{ Year } & \multicolumn{5}{|c||}{ Million Pounds of Active Ingredient } \\
\cline { 2 - 6 } & Herbicides/PGR & Insecticides & Fungicides & Other & Total \\
\hline 2001 & 433 & 73 & 42 & 359 & 907 \\
\hline 2000 & 432 & 90 & 44 & 382 & 948 \\
\hline 1999 & 428 & 93 & 45 & 390 & 956 \\
\hline 1998 & 465 & 69 & 54 & 348 & 936 \\
\hline 1997 & 470 & 79 & 53 & 353 & 955 \\
\hline 1996 & 481 & 81 & 51 & 342 & 955 \\
\hline 1995 & 461 & 85 & 49 & 338 & 933 \\
\hline 1994 & 485 & 80 & 48 & 326 & 939 \\
\hline 1993 & 425 & 72 & 47 & 320 & 864 \\
\hline 1992 & 450 & 78 & 45 & 311 & 884 \\
\hline 1991 & 440 & 77 & 47 & 284 & 848 \\
\hline 1990 & 455 & 82 & 50 & 297 & 884 \\
\hline 1989 & 460 & 85 & 54 & 274 & 873 \\
\hline 1988 & 450 & 91 & 54 & 272 & 867 \\
\hline 1987 & 425 & 98 & 52 & 271 & 846 \\
\hline 1986 & 481 & 105 & 59 & 282 & 927 \\
\hline 1985 & 501 & 113 & 59 & 288 & 961 \\
\hline 1984 & 516 & 122 & 56 & 294 & 988 \\
\hline 1983 & 455 & 131 & 59 & 296 & 941 \\
\hline 1982 & 503 & 141 & 59 & 308 & 1011 \\
\hline \hline
\end{tabular}

Table 7. U.S. pesticide amounts used, industry/commercial/government sector.

\begin{tabular}{||c|c|c|c|c|c||}
\hline \hline \multirow{2}{*}{ Year } & \multicolumn{5}{|c||}{ Million Pounds of Active Ingredient } \\
\cline { 2 - 6 } & Herbicides/PGR & Insecticides & Fungicides & Other & Total \\
\hline 2001 & 49 & 15 & 19 & 50 & 133 \\
\hline 2000 & 48 & 17 & 19 & 52 & 136 \\
\hline 1999 & 52 & 19 & 24 & 53 & 148 \\
\hline 1998 & 41 & 21 & 24 & 52 & 138 \\
\hline 1997 & 49 & 20 & 52 & 141 \\
\hline 1996 & 49 & 24 & 20 & 52 & 145 \\
\hline 1995 & 48 & 28 & 53 & 149 \\
\hline 1994 & 52 & 30 & 23 & 54 & 159 \\
\hline 1993 & 56 & 30 & 25 & 56 & 167 \\
\hline 1992 & 58 & 27 & 28 & 57 & 170 \\
\hline 1991 & 60 & 26 & 30 & 58 & 174 \\
\hline 1990 & 63 & 27 & 31 & 60 & 181 \\
\hline 1989 & 63 & 27 & 31 & 60 & 181 \\
\hline 1988 & 64 & 27 & 32 & 61 & 184 \\
\hline 1987 & 65 & 28 & 34 & 61 & 188 \\
\hline 1986 & 68 & 29 & 36 & 64 & 197 \\
\hline 1985 & 70 & 30 & 37 & 64 & 201 \\
\hline 1984 & 78 & 31 & 38 & 65 & 212 \\
\hline \hline
\end{tabular}


Pesticide Use Trends in the U.S.: a 20-Year U.S. Summary

Table 8. U.S. pesticide amounts used, home and garden sector.

\begin{tabular}{||c|c|c|c|c|c||}
\hline \hline \multirow{2}{*}{ Year } & \multicolumn{5}{|c||}{ Million Pounds of Active Ingredient } \\
\cline { 2 - 6 } & Herbicides/PGR & Insecticides & Fungicides & Other & Total \\
\hline 2001 & 71 & 17 & 12 & 63 & 163 \\
\hline 2000 & 62 & 15 & 11 & 62 & 150 \\
\hline 1999 & 54 & 14 & 10 & 62 & 140 \\
\hline 1998 & 49 & 13 & 8 & 62 & 132 \\
\hline 1997 & 49 & 13 & 62 & 132 \\
\hline 1996 & 48 & 12 & 8 & 62 & 130 \\
\hline 1995 & 47 & 12 & 8 & 61 & 128 \\
\hline 1994 & 46 & 13 & 8 & 63 & 130 \\
\hline 1993 & 46 & 13 & 8 & 64 & 131 \\
\hline 1992 & 46 & 12 & 8 & 66 & 132 \\
\hline 1991 & 46 & 12 & 9 & 67 & 134 \\
\hline 1990 & 46 & 12 & 10 & 68 & 136 \\
\hline 1989 & 44 & 12 & 13 & 70 & 139 \\
\hline 1988 & 43 & 13 & 13 & 70 & 139 \\
\hline 1987 & 42 & 14 & 14 & 70 & 140 \\
\hline 1986 & 41 & 16 & 14 & 70 & 141 \\
\hline 1985 & 40 & 18 & 14 & 70 & 142 \\
\hline 1984 & 40 & 20 & 15 & 70 & 145 \\
\hline 1983 & 38 & 22 & 16 & 70 & 146 \\
\hline 1982 & 37 & 24 & 17 & 70 & 148 \\
\hline \hline
\end{tabular}

